OPEN ACCESS

Edited by:

Kan Gong,

Peking University First Hospital, China

Reviewed by:

Yun Guan,

Johns Hopkins Medicine,

United States

Enrico Checcucci,

Istituto di Candiolo (IRCCS), Italy

${ }^{*}$ Correspondence:

Ning Zhang

niru7429@126.com

Ming LiU

liuming19731029@163.com

Yuan Li

yuanlixy@csu.edu.cn

Xiongjun $Y_{e}$

yexiongjun@bjmu.edu.cn

Wanhai Xu

xuwanhai@hrbmu.edu.cn

Specialty section:

This article was submitted to

Genitourinary Oncology,

a section of the journal

Frontiers in Oncology

Received: 05 August 2021

Accepted: 31 August 2021 Published: 21 September 2021

Citation:

Hong B, Hou H, Chen L, Li Z, Zhang Z, Zhao Q, Du X, Li Y, Ye X,

Xu W, Liu M and Zhang N (2021)

The Clinicopathological Features and Prognosis in Patients With

Papillary Renal Cell Carcinoma:

A Multicenter Retrospective

Study in Chinese Population.

Front. Oncol. 11:753690.

doi: 10.3389/fonc.2021.753690

\section{The Clinicopathological Features and Prognosis in Patients With Papillary Renal Cell Carcinoma: A Multicenter Retrospective Study in Chinese Population}

\author{
Baoan Hong ${ }^{1}$, Huimin Hou ${ }^{2}$, Lingxiao $\mathrm{Chen}^{3}$, Zhi Li ${ }^{3}$, Zhipeng Zhang ${ }^{2}$, Qiang Zhao ${ }^{1}$, \\ Xin $\mathrm{Du}^{1}$, Yuan $\mathrm{Li}^{3,4 *}$, Xiongjun $\mathrm{Ye}^{5 *}$, Wanhai $\mathrm{Xu}^{6 *}$, Ming $\mathrm{Liu}^{2 *}$ and Ning Zhang ${ }^{1 *}$ \\ ${ }^{1}$ Key Laboratory of Carcinogenesis and Translational Research (Ministry of Education/Beijing), Department of Urology, \\ Peking University Cancer Hospital \& Institute, Beijing, China, ${ }^{2}$ Department of Urology, Beijing Hospital, National Center of \\ Gerontology, Institute of Geriatric Medicine, Chinese Academy of Medical Sciences, Beijing, China, ${ }^{3}$ Department of Urology, \\ Xiangya Hospital, Central South University, Changsha, China, ${ }^{4}$ Department of Urology, The Second Xiangya Hospital, \\ Central South University, Changsha, China, ${ }^{5}$ Urology and Lithotripsy Center, Peking University People's Hospital, \\ Beijing, China, ${ }^{6}$ Department of Urology, The 4th Affiliated Hospital of Harbin Medical University, Harbin, China
}

Objective: The purpose of this study was to compare the clinicopathological characteristics of type 1 and type 2 papillary renal cell carcinoma (PRCC) and to explore the prognostic factors of PRCC in the Chinese population.

Methods: A total of 242 patients with PRCC from five Chinese medical centers were retrospectively included. From them, 82 were type 1 PRCC and 160 were type 2 PRCC. Clinicopathological features and oncologic outcomes were reviewed. The Kaplan-Meier analysis and log-rank test were performed to describe the progression-free survival (PFS) and overall survival (OS). Univariate and multivariate Cox proportional hazards regression models were used to analyze the prognostic factors of PRCC.

Results: Of the 242 patients, the average age at surgery was $55.3 \pm 13.1$ years. The mean tumor size was $5.1 \pm 3.1 \mathrm{~cm}$. Compared with type 1 PRCC patients, type 2 PRCC patients had a larger tumor size and were more likely to undergo radical nephrectomy. Besides, type 2 PRCC patients had higher tumor stage $(p<0.001)$ and WHO International Society of Urological Pathology (WHO/ISUP) grading $(p<0.001)$. Furthermore, tumor necrosis was more common in type 2 PRCC than type 1 PRCC $(p=0.030)$. The Kaplan-Meier survival analysis showed that the PFS and OS of type 1 PRCC patients were significantly better than those of type 2 PRCC patients ( $p=0.0032$ and $p=0.0385$, respectively). Univariate analysis showed that tumor size, surgical procedures, pT stage, WHO/ISUP grading, and microvascular invasion were significant predictors of PFS and OS for type 2 PRCC patients. In the multivariate analysis, only pT stage $(p=0.004)$ and WHO/ISUP grading $(p=0.010$ ) were the independent risk factors. Among type 2 PRCC patients with $\mathrm{pT} 1$ stage, no significant difference was found in PFS and OS between the partial nephrectomy and radical nephrectomy groups ( $p=0.159$ and $p=0.239$, respectively). 
Conclusion: This multi-institutional study reveals the significant differences in clinicopathological variables and oncologic outcomes between type 1 and 2 PRCC. For type 2 PRCC in PT1 stage, the prognosis of partial nephrectomy is not inferior to that of radical nephrectomy, and nephron-sparing surgery can be considered.

Keywords: carcinoma, papillary renal cell carcinoma, clinical features, pathological features, prognosis

\section{BACKGROUND}

Papillary renal cell carcinoma (PRCC) is one of the most common renal cell carcinoma (RCC), second only to clear cell RCC, accounting for $15 \%$ to $20 \%$ of all RCCs (1). It has significant heterogeneity, mainly for the different histopathological subtypes, biological behaviors, and clinical outcomes. The initial study by Delahunt and Eble reported that PRCC can be categorized into type 1 and type 2 based on histomorphological and immunohistochemical characteristics (2). Typically, type 1 tumor cells are cubic, are basophilic in cytoplasm, and have small nuclei, and the papillary structure is covered by a single layer of cells; while type 2 tumor cells are tall columnar, are rich in eosinophilic cytoplasm, and have obvious nucleoli, and the papillary structure is covered by pseudostratified cells (3).

According to previous studies, the two PRCC subtypes have differences in clinicopathological characteristics and prognosis. PRCC type 2 has a more advanced stage, a higher nuclear grade, and a worse prognosis than type 1 (4-6). However, on the contrary, some studies reported that PRCC subtyping had no effect on oncologic outcomes (7-10). Therefore, whether the histologic subtypes affect the prognosis are still controversial. In addition, most of these studies were carried out in Western populations. There is still a lack of studies to reveal the different clinical significance between PRCC subtypes in the Chinese population.

Hence, in the present study, we conducted a multicenter retrospective study that included a large sample of Chinese PRCC patients. The goal was to better understand the clinicopathological features and clinical outcomes of type 1 and type 2 PRCC in the Chinese population, further reveal prognostic factors, and provide clinical guidance.

\section{METHODS}

\section{Patient Population and Clinicopathological Features}

Under the supervision of the Institutional Review Board, the data of RCC patients who underwent radical nephrectomy (RN) or partial nephrectomy $(\mathrm{PN})$ at five medical centers in China from 2010 to 2020 were reviewed. Patients with incomplete clinicopathological data or lost to follow-up were eliminated. A total of 242 patients pathologically diagnosed with PRCC were included. Of these patients definite in classification, 82 patients were type 1 PRCC and 160 cases were type 2.

Clinical features included patient gender, age, initial symptoms, tumor location, and surgical procedures. Pathological parameters included PRCC subtypes, tumor size, pathologic T (pT) stage, grade, microvascular invasion, necrosis, and sarcomatoid differentiation. The samples were graded according to 2016 WHO International Society of Urological Pathology (WHO/ISUP) grading system. The pathological staging was determined according to the 2018 American Joint Committee on Cancer staging manual.

The main outcomes concerned were progression-free survival (PFS) and overall survival (OS). In this study, PFS was defined as the duration from surgery to local recurrence or distant metastasis, and OS was defined as the duration from surgery to death from any cause. Surviving patients were censored at the last follow-up.

\section{Statistical Analysis}

The independent samples $t$-test was used to compare the continuous variables, and the $\chi^{2}$ test was used to compare the categorical variables. The impacts of the PRCC subclassification and clinicopathological features on PFS and OS were described with the Kaplan-Meier curve and compared with the log-rank test. Associations of clinical and pathological features with PFS and OS were analyzed using univariate and multivariate Cox proportional hazards regression models and presented with the HR and 95\% CI. Ten variables were included in the stepwise selection analysis. Statistical analysis was performed with SPSS version 22.0 (IBM Corporation, Armonk, NY), and two-sided $p<$ 0.05 was defined as statistically significant.

\section{RESULTS}

In this study, 177 (73.1\%) of the patients were male and 65 (26.9\%) of them were female. The male-to-female ratio was 2.7 to 1 . The average age at surgery was $55.3 \pm 13.1$ years (ranging from 15 to 82 ). Of the 242 patients, $68.6 \%$ of patients were asymptomatic and were detected incidentally through physical examination. The clinical symptoms of the remaining cases included flank pain (14.5\%), hematuria (12.0\%), and other symptoms such as fever or renal dysfunction (5.0\%). The mean tumor size was $5.1 \pm 3.1 \mathrm{~cm}$. The pathologic stage was T1a in 111 cases $(45.9 \%), \mathrm{T} 1 \mathrm{~b}$ in 62 (25.6\%), T2 in 35 (14.5\%), T3 in 22 (9.1\%), and T4 in 12 (5.0\%). The WHO/ISUP grading was G1 in 35 cases (14.5\%), G2 in 98 (40.5\%), G3 in 91 (37.6\%), and G4 in 18 (7.4\%).

The baseline clinicopathological characteristics of the PRCC patients are listed in Table 1. As shown in the table, RN was more common for type 2 PRCC patients than for type 1 patients (70.6\% vs. 32.9\%; $p<0.001$ ). Compared with type 1 PRCC tumors, type 2 PRCC tumors were larger $(p<0.001)$. Besides, type 2 PRCC patients were likely to have a more advanced tumor 
stage $(p<0.001)$ and higher WHO/ISUP grade $(p<0.001)$. Moreover, the incidence of tumor necrosis in type 2 PRCC is higher than that in type $1 \operatorname{PRCC}(p=0.030)$. Other clinicopathological characteristics (including gender, age, initial symptoms, tumor location, microvascular invasion, and sarcomatoid differentiation) had no significant differences between the two subtypes.

The average follow-up duration was 48.9 months. To the end of follow-up, a total of 31 patients underwent disease progression, and 22 cases had died. The Kaplan-Meier plots for PFS indicated that type 2 PRCC patients had a lower probability for PFS than type 1 PRCC ( $p=0.0032$, Figure 1A). The estimated 1-year PFS for type 1 and type 2 PRCC patients reached $98.8 \%$ and $91.9 \%$, respectively; and their 5-year PFS was $95.5 \%$ and $81.3 \%$, respectively. In addition, the risk of death in patients with type 2 PRCC is significantly higher than that of patients with type 1 PRCC ( $p=0.0385$, Figure 1B). The 1 -year OS of type 1 and type 2 PRCC reached $98.8 \%$ and $96.2 \%$, respectively; and their 5-year OS was $95.3 \%$ and $85.9 \%$. Thus, the Kaplan-Meier survival analysis demonstrated that the PFS

TABLE 1 | Comparison of clinicopathological characteristics between type 1 and type 2 PRCC.

\begin{tabular}{|c|c|c|c|}
\hline Characteristic & Type $1(n=82)$ & Type $2(n=160)$ & $p$ \\
\hline Gender & & & 0.535 \\
\hline Male & 62 & 115 & \\
\hline Female & 20 & 45 & \\
\hline Age (years) & $55.3 \pm 11.7$ & $55.2 \pm 13.8$ & 0.988 \\
\hline Initial symptoms & & & 0.154 \\
\hline No symptoms & 63 & 103 & \\
\hline Hematuria & 5 & 24 & \\
\hline Lumbago & 10 & 25 & \\
\hline Other & 4 & 8 & \\
\hline Tumor location & & & 0.782 \\
\hline Left & 41 & 77 & \\
\hline Right & 41 & 83 & \\
\hline Surgical procedures & & & $<0.001$ \\
\hline Partial nephrectomy & 55 & 47 & \\
\hline Radical nephrectomy & 27 & 113 & \\
\hline Tumor size (cm) & $4.1 \pm 2.5$ & $5.6 \pm 3.3$ & $<0.001$ \\
\hline Pathologic T stage & & & $<0.001$ \\
\hline T1a & 55 & 56 & \\
\hline T1b & 13 & 49 & \\
\hline T2 & 10 & 25 & \\
\hline T3 & 4 & 18 & \\
\hline T4 & 0 & 12 & \\
\hline WHO/ISUP grade & & & $<0.001$ \\
\hline G1 & 25 & 10 & \\
\hline G2 & 36 & 62 & \\
\hline G3 & 17 & 74 & \\
\hline G4 & 4 & 14 & \\
\hline Microvascular invasion & & & 0.179 \\
\hline No & 77 & 141 & \\
\hline Yes & 5 & 19 & \\
\hline Tumor necrosis & & & 0.030 \\
\hline No & 68 & 111 & \\
\hline Yes & 14 & 49 & \\
\hline Sarcomatoid differentiation & & & 0.665 \\
\hline No & 81 & 156 & \\
\hline Yes & 1 & 4 & \\
\hline
\end{tabular}

WHO/ISUP, WHO International Society of Urological Pathology. and OS of type 1 PRCC were significantly better than those of type 2 PRCC patients.

Given the fact that statistical power was weakened by the limited progression or death events among type 1 PRCC, a subgroup univariate and multivariate Cox regression analysis of factors predictive of PFS and OS in type 2 PRCC patients was further performed. Univariate analysis showed that tumor size $(p=0.015)$, surgical procedures $(p=0.010)$, pT stage $(p<0.001)$, WHO/ISUP grading $(p<0.001)$, and microvascular invasion $(p=$ 0.001) were significantly associated with PFS (Table 2). However, gender, age, tumor location, tumor necrosis, and sarcomatoid differentiation had no significant influence on PFS. According to multivariate analysis, only pT stage $(p=$ $0.004)$ and $\mathrm{WHO} / \mathrm{ISUP}$ grading $(p=0.010)$ were the independent risk factors for PFS of type 2 PRCC patients (Table 2). In univariate analysis, tumor size, surgical procedures, pT stage, WHO/ISUP grading, and microvascular invasion were significant predictors of OS (Table 3 ). Furthermore, stepwise multivariate analysis showed that $\mathrm{pT}$ stage $(p=0.007)$ and WHO/ISUP grading $(p=0.031)$ were independent predictors of OS (Table 3). Overall, of the various significant variables in univariate analysis, only $\mathrm{pT}$ stage and WHO/ISUP grading were independent risk factors for both PFS and $O S$ in multivariate analysis.

Of the 160 type 2 PRCC patients, 57 patients received $\mathrm{PN}$ and 103 cases received RN. The pathologic stage was $\mathrm{T} 1$ in 110 cases (68.8\%), T2 in $22(13.8 \%)$, T3 in $16(10.0 \%)$, and $\mathrm{T} 4$ in $12(7.5 \%)$. The average follow-up duration was 47.9 months. For type 2 PRCC patients with pT1 stage, stratified analysis showed that no significant difference in PFS ( $p=0.159$, Figure 1C) and OS ( $p=$ 0.239 , Figure 1D) was found between $\mathrm{PN}$ and $\mathrm{RN}$ groups.

\section{DISCUSSION}

PRCC accounts for the largest subset of non-clear cell RCC, while its overall incidence is not high (11). At present, there is still a lack of multicenter large-scale study about PRCC, and further exploration of the clinicopathological features and prognosis of PRCC is helpful for understanding this type of RCC. In 1976, Mancilla-Jimenez et al. (12) retrospectively analyzed the clinicopathological data of 224 cases of RCC including 34 cases of PRCC and proposed it as an independent subtype of RCC. In 1997, Delahunt and Eble (2) classified PRCC into two morphologically different types according to cytological structural characteristics. As reported, type 1 PRCC is more common than type 2 PRCC in Western population with a 2 3:1 ratio (13-15). However, in this study, the distribution between type 1 and type 2 PRCC was approximately 1:2, which was contrary to that reported in Western population. On the other hand, Ha et al. (16) reported a multi-institutional study of 274 Korean patients with PRCC, of which 118 had type 1 PRCC and 156 had type 2 PRCC. Thus, the high incidence of type 2 PRCC may be a distinct characteristic of the Asian population.

As reported, PRCC accounted for approximately $10 \%-14 \%$ of RCC in Western population. However, the incidence of PRCC 

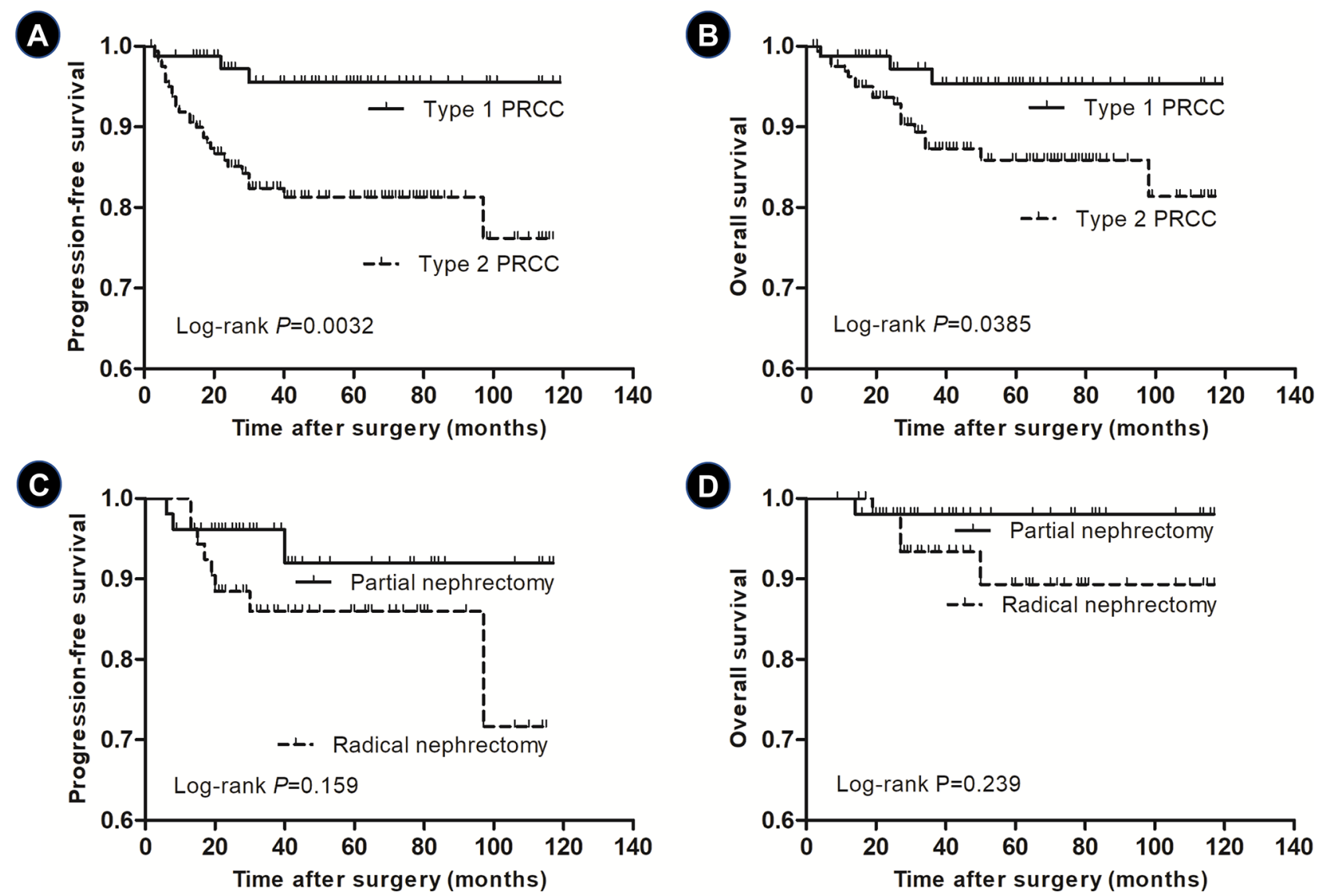

FIGURE 1 | (A) Comparison of progression-free survival (PFS) between type 1 and type 2 papillary renal cell carcinoma (PRCC) ( $p=0.0032)$. (B) Comparison of overall survival (OS) between type 1 and type 2 PRCC $(p=0.0385)$. (C) Comparison of PFS of type 2 PRCC patients with pT1 stage receiving partial nephrectomy and radical nephrectomy $(p=0.159)$. (D) Comparison of OS of type 2 PRCC patients with $\mathrm{pT} 1$ stage receiving partial nephrectomy and radical nephrectomy $(p=0.239)$.

TABLE 2 | Univariate and multivariate Cox regression analyses of factors predictive of progression-free survival in type 2 PRCC patients.

\begin{tabular}{|c|c|c|c|c|c|c|}
\hline Variable & \multicolumn{3}{|c|}{ Univariate analysis } & \multicolumn{3}{|c|}{ Multivariate analysis } \\
\hline Age (>55 vs. $\leq 55)$ & 1.582 & $0.728-3.437$ & 0.246 & - & - & - \\
\hline Tumor location (left vs. right) & 1.505 & $0.712-3.182$ & 0.285 & - & - & - \\
\hline Tumor size $(>5.8 \mathrm{~cm}$ vs. $\leq 5.8 \mathrm{~cm})$ & 2.617 & $1.205-5.683$ & 0.015 & 1.032 & $0.450-2.364$ & 0.941 \\
\hline Surgical procedures (RN vs. PN) & 4.877 & $1.472-16.160$ & 0.010 & 2.562 & $0.713-9.210$ & 0.149 \\
\hline Microvascular invasion (yes vs. no) & 4.138 & $1.820-9.408$ & 0.001 & 1.356 & $0.496-3.709$ & 0.553 \\
\hline Tumor necrosis (yes vs. no) & 1.095 & $0.495-2.421$ & 0.823 & - & - & - \\
\hline Sarcomatoid differentiation (yes vs. no) & 2.186 & $0.296-16.171$ & 0.444 & - & - & - \\
\hline
\end{tabular}

PRCC, papillary renal cell carcinoma; RN, radical nephrectomy; PN, partial nephrectomy; WHO/ISUP, WHO International Society of Urological Pathology.

was reported to be approximately $5.2 \%-5.6 \%$ in Japanese and Korean populations and $1.9-7.5 \%$ in Chinese population $(5,16,17)$. In this study, PRCC accounted for $4.3 \%$ of RCC, which was significantly lower than the incidence reported in the West, but a similar incidence was reported in Japan, Korea, and China.
However, whether the low incidence of PRCC is a characteristic of Asian population deserves further investigation. In terms of clinical symptoms at first diagnosis, most of patients in this study were asymptomatic (68.6\%). Patients who presented symptoms accounted for $31.4 \%$, and type 2 PRCC patients were more 
TABLE 3 | Univariate and multivariate Cox regression analyses of factors predictive of overall survival in type 2 PRCC patients.

\begin{tabular}{|c|c|c|c|c|c|c|}
\hline \multirow[t]{2}{*}{ Variable } & \multicolumn{3}{|c|}{ Univariate analysis } & \multicolumn{3}{|c|}{ Multivariate analysis } \\
\hline & HR & $95 \% \mathrm{Cl}$ & $p$ & HR & $95 \% \mathrm{Cl}$ & $p$ \\
\hline Gender (male vs. female) & 1.014 & $0.364-2.821$ & 0.979 & - & - & - \\
\hline Age (>55 vs. $\leq 55)$ & 1.077 & $0.430-2.695$ & 0.875 & - & - & - \\
\hline Tumor location (left vs. right) & 1.255 & $0.510-3.092$ & 0.621 & - & - & - \\
\hline Tumor size (>5.8 cm vs. $\leq 5.8 \mathrm{~cm}$ ) & 5.224 & $1.732-15.753$ & 0.003 & 1.706 & $0.534-5.451$ & 0.368 \\
\hline Surgical procedures (RN vs. PN) & 9.531 & $1.272-71.431$ & 0.028 & 3.346 & $0.403-27.754$ & 0.263 \\
\hline T stage (III/IV vs. I/II) & 8.425 & 3.305-21.479 & $<0.001$ & 4.489 & $1.506-13.382$ & 0.007 \\
\hline WHO/ISUP grade (G3/4 vs. G1/2) & 18.848 & $2.508-141.618$ & 0.004 & 9.701 & $1.235-76.171$ & 0.031 \\
\hline Microvascular invasion (yes vs. no) & 4.225 & $1.602-11.142$ & 0.004 & 1.671 & $0.542-5.148$ & 0.371 \\
\hline Tumor necrosis (yes vs. no) & 1.072 & $0.407-2.823$ & 0.888 & - & - & - \\
\hline Sarcomatoid differentiation (yes vs. no) & 4.410 & $0.575-33.809$ & 0.153 & - & - & - \\
\hline
\end{tabular}

PRCC, papillary renal cell carcinoma; RN, radical nephrectomy; PN, partial nephrectomy; WHO/ISUP, WHO International Society of Urological Pathology.

common. Type 2 PRCC is highly aggressive and prone to progression, often with early symptoms such as hematuria and flank pain.

In this PRCC cohort, patients with pT1 account for $71.5 \%$, and patients with G2/G3 account for 78.1\%. Moreover, studies had reported that PRCC patients with pT1 account for $70 \%-$ $80 \%$, and those with G2 and G3 accounted for 36\%-43\% and $32 \%-51 \%$, respectively $(13,16)$. Therefore, the pathological staging of PRCC is mostly early stage, mainly T1 stage, and majority of the pathological grades are grade 2 and grade 3. Earlier studies indicated that prognosis between the two subclassifications of PRCC was different. Pignot et al. (4) reported that type 2 PRCC was associated with a later stage and a higher grade, and the incidence of microvascular invasion was higher $(p<0.001)$. Delahunt and Eble summarized that type 2 PRCC had a higher stage and grade than type 1, which was associated with worse prognosis (2). In this study, we revealed that type 2 patients had a more advanced $\mathrm{pT}$ stage and higher WHO/ISUP grading $(p<0.001)$ than had type 1 . These pathological features of type 2 PRCC suggest that its tumor biological behavior may be more aggressive.

At present, the issue about the prognostic factors of PRCC patients is still controversial. For example, Pignot et al. (4) reported that tumor type, stage, grade, microvascular invasion, an absence of foam cells, the presence of sarcomatoid cells, and tumor necrosis were prognostic factors of PRCC, of which tumor type and TNM stage were independent prognostic factors. However, Ha et al. (16) demonstrated that subclassification of PRCC was not a significant prognostic factor, while the pathologic $\mathrm{T}$ stage was an independent prognostic factor. Some studies reported that the biological behavior of type 2 PRCC is more aggressive than type 1, whereas others claimed that subclassification of PRCC did not affect the prognosis. In this study, the 1-year OS of type 1 and type 2 PRCC reached $98.8 \%$ and $96.2 \%$, respectively, while the 5-year OS were $95.3 \%$ and $85.9 \%$, indicating that the prognosis of type 2 was worse than that of type 1 . Further univariate and multivariate analyses for type 2 PRCC indicated that tumor size, surgical procedures, $\mathrm{pT}$ stage, WHO/ISUP grading, and microvascular invasion were significant predictors of OS, while only the pT stage and $\mathrm{WHO} /$ ISUP grading were independent predictors of OS.
The surgical procedures play an important role in clinical treatment decisions. It was reported that nephron-sparing surgery is recommended as a priority for PRCC, and RN is preferable when nephron-saving surgery is not appropriate (18). Histologically, type 2 PRCC is more aggressive than type 1 PRCC, with higher pathological stage and grade, which may account for $\mathrm{RN}$ being recommended for type 2 PRCC patients. However, Bigot et al. (7) showed that histological subtyping had no impact on oncologic outcomes for PRCC patients receiving nephron-sparing surgery, which is suitable for localized PRCC. In the present study, stratified analysis showed that no significant difference in PFS ( $p=$ $0.159)$ and $\mathrm{OS}(p=0.239)$ was found between $\mathrm{PN}$ and $\mathrm{RN}$ groups for type 2 PRCC patients with pT1 stage. As the study was retrospective, for tumors of the same stage, surgeons might choose different surgical methods according to the tumor location and the patient's condition, which might lead to analytical bias. However, during the limited follow-up duration in this study, the prognosis of pT1 patients with type 2 PRCC who underwent PN was not worse than that of RN. The results may suggest that multifocality is not common in low-stage type 2 PRCC and PN could be considered for patients with stage T1 who are suspected to be type 2 PRCC preoperatively.

Although this is a multi-institutional study on Chinese PRCC patients, potential limitations need to be noticed. It is a retrospective study, and the follow-up duration was limited, so the strength of evidence needs to be further improved. Additionally, patients who did not have access to complete clinicopathological data were excluded, which might lead to selection bias. Moreover, the preferences and experience of surgeons and pathologists are difficult to adjust, which can also cause bias.

\section{CONCLUSIONS}

Our findings indicated that the PFS and OS of type 1 PRCC were significantly better than those of type 2 PRCC. Compared with type 1 PRCC, type 2 PRCC had higher tumor stage and WHO/ ISUP grading. Furthermore, tumor necrosis was more common in type 2 PRCC than type 1 . In multivariate analysis of type 2 PRCC, pT stage and WHO/ISUP grading were independent predictors of PFS and OS. Interestingly, among type 2 PRCC 
with pT1 stage, no significant difference was observed in PFS and OS between $\mathrm{PN}$ and $\mathrm{RN}$ groups.

\section{DATA AVAILABILITY STATEMENT}

The raw data supporting the conclusions of this article will be made available by the authors, without undue reservation.

\section{ETHICS STATEMENT}

The studies involving human participants were reviewed and approved by Institutional Review Board of Peking University Cancer Hospital \& Institute. The patients/participants provided their written informed consent to participate in this study.

\section{AUTHOR CONTRIBUTIONS}

Conceptualization: NZ, ML, YL, WX, and XY. Data curation: $\mathrm{BH}$, $\mathrm{HH}, \mathrm{LC}, \mathrm{ZL}, \mathrm{ZZ}, \mathrm{QZ}$, and XD. Formal analysis: BH, HH, LC, ZL,

\section{REFERENCES}

1. Akhtar M, Al-Bozom IA, Al HT. Papillary Renal Cell Carcinoma (PRCC): An Update. Adv Anat Pathol (2019) 26(2):124-32. doi: 10.1097/PAP. 0000000000000220

2. Delahunt B, Eble JN. Papillary Renal Cell Carcinoma: A Clinicopathologic and Immunohistochemical Study of 105 Tumors. Mod Pathol (1997) 10 (6):537-44

3. Jiang F, Richter J, Schraml P, Bubendorf L, Gasser T, Sauter G, et al. Chromosomal Imbalances in Papillary Renal Cell Carcinoma: Genetic Differences Between Histological Subtypes. Am J Pathol (1998) 153 (5):1467-73. doi: 10.1016/S0002-9440(10)65734-3

4. Pignot G, Elie C, Conquy S, Vieillefond A, Flam T, Zerbib M, et al. Survival Analysis of 130 Patients With Papillary Renal Cell Carcinoma: Prognostic Utility of Type 1 and Type 2 Subclassification. Urology (2007) 69(2):230-5. doi: 10.1016/j.urology.2006.09.052

5. Yamashita S, Ioritani N, Oikawa K, Aizawa M, Endoh M, Arai Y. Morphological Subtyping of Papillary Renal Cell Carcinoma: Clinicopathological Characteristics and Prognosis. Int J Urol (2007) 14(8):679-83. doi: 10.1111/j.14422042.2007.01805.x

6. Wong E, Di Lena R, Breau RH, Pouliot F, Finelli A, Lavallee LT, et al. Morphologic Subtyping as a Prognostic Predictor for Survival in Papillary Renal Cell Carcinoma: Type 1 vs. Type 2. Urol Oncol (2019) 37(10):721-6. doi: 10.1016/j.urolonc.2019.05.009

7. Bigot P, Bernhard JC, Gill IS, Vuong NS, Verhoest G, Flamand V, et al. The Subclassification of Papillary Renal Cell Carcinoma Does Not Affect Oncological Outcomes After Nephron Sparing Surgery. World J Urol (2016) 34(3):347-52. doi: 10.1007/s00345-015-1634-0

8. Yamanaka K, Miyake H, Hara I, Inoue TA, Hanioka K, Fujisawa M. Papillary Renal Cell Carcinoma: A Clinicopathological Study of 35 Cases. Int J Urol (2006) 13(8):1049-52. doi: 10.1111/j.1442-2042.2006.01500.x

9. Le X, Wang XB, Zhao H, Chen RF, Ge P. Comparison of Clinicopathologic Parameters and Oncologic Outcomes Between Type 1 and Type 2 Papillary Renal Cell Carcinoma. BMC Urol (2020) 20(1):148. doi: 10.1186/s12894-02000716-0

10. Yang C, Shuch B, Kluger H, Humphrey PA, Adeniran AJ. High WHO/ISUP Grade and Unfavorable Architecture, Rather Than Typing of Papillary Renal Cell Carcinoma, May Be Associated With Worse Prognosis. Am J Surg Pathol (2020) 44(5):582-93. doi: 10.1097/PAS.0000000000001455
ZZ, QZ, and XD. Funding acquisition: NZ, ML, and YL. Project administration and supervision: NZ, ML, YL, WX, and XY. Original draft writing: $\mathrm{BH}$ and NZ. All authors contributed to the article and approved the submitted version.

\section{FUNDING}

This study was supported by Capital's Funds for Health Improvement and Research (Grant No. 2020-2-1024); Capital Clinical Characteristic Application Research Project (Grant No. Z171100001017201); the Natural Science Foundation of Beijing (Grant No. 7212010); and Beijing Xisike Clinical Oncology Research Foundation (Grant No. Y-2019AZZD-0369).

\section{ACKNOWLEDGMENTS}

We sincerely thank all the patients involved in this study for their collaboration and support. We acknowledge Jia Xue from Crown Bioscience for her assistance in language editing.

11. Schrader AJ, Rauer-Bruening S, Olbert PJ, Hegele A, Rustemeier J, Timmesfeld N, et al. Incidence and Long-Term Prognosis of Papillary Renal Cell Carcinoma. J Cancer Res Clin Oncol (2009) 135(6):799-805. doi: 10.1007/s00432-008-0515-y

12. Mancilla-Jimenez R, Stanley RJ, Blath RA. Papillary Renal Cell Carcinoma: A Clinical, Radiologic, and Pathologic Study of 34 Cases. Cancer (1976) 38 (6):2469-80. doi: 10.1002/1097-0142(197612)38:6<2469::AID CNCR2820380636>3.0.CO;2-R

13. Cornejo KM, Dong F, Zhou AG, Wu CL, Young RH, Braaten K, et al. Papillary Renal Cell Carcinoma: Correlation of Tumor Grade and Histologic Characteristics With Clinical Outcome. Hum Pathol (2015) 46(10):1411-7. doi: 10.1016/j.humpath.2015.07.001

14. Sukov WR, Lohse CM, Leibovich BC, Thompson RH, Cheville JC. Clinical and Pathological Features Associated With Prognosis in Patients With Papillary Renal Cell Carcinoma. J Urol (2012) 187(1):54-9. doi: 10.1016/ j.juro.2011.09.053

15. Polifka I, Agaimy A, Herrmann E, Spath V, Trojan L, Stockle M, et al. High Proliferation Rate and TNM Stage But Not Histomorphological Subtype are Independent Prognostic Markers for Overall Survival in Papillary Renal Cell Carcinoma. Hum Pathol (2019) 83:212-23. doi: 10.1016/j.humpath.2018.08.006

16. Ha YS, Chung JW, Choi SH, Lee JN, Kim HT, Kim TH, et al. Clinical Significance of Subclassification of Papillary Renal Cell Carcinoma: Comparison of Clinicopathologic Parameters and Oncologic Outcomes Between Papillary Histologic Subtypes 1 and 2 Using the Korean Renal Cell Carcinoma Database. Clin Genitourin Cancer (2017) 15(2):e181-6. doi: 10.1016/j.clgc.2016.07.020

17. Pan H, Ye L, Zhu Q, Yang Z, Hu M. The Effect of the Papillary Renal Cell Carcinoma Subtype on Oncological Outcomes. Sci Rep (2020) 10(1):21073. doi: 10.1038/s41598-020-78174-9

18. Fernandes DS, Lopes JM. Pathology, Therapy and Prognosis of Papillary Renal Carcinoma. Future Oncol (2015) 11(1):121-32. doi: 10.2217/fon.14.133

Conflict of Interest: The authors declare that the research was conducted in the absence of any commercial or financial relationships that could be construed as a potential conflict of interest.

Publisher's Note: All claims expressed in this article are solely those of the authors and do not necessarily represent those of their affiliated organizations, or those of the publisher, the editors and the reviewers. Any product that may be evaluated in 
this article, or claim that may be made by its manufacturer, is not guaranteed or endorsed by the publisher.

Copyright $\odot 2021$ Hong, Hou, Chen, Li, Zhang, Zhao, Du, Li, Ye, Xu, Liu and Zhang. This is an open-access article distributed under the terms of the Creative Commons
Attribution License (CC BY). The use, distribution or reproduction in other forums is permitted, provided the original author $(s)$ and the copyright owner(s) are credited and that the original publication in this journal is cited, in accordance with accepted academic practice. No use, distribution or reproduction is permitted which does not comply with these terms. 